\title{
Caractérisation de quelques sols majeurs de la Sologne centrale. Rôle des facteurs écologiques dans la répartition et l'évolution des profils
}

\author{
Ildud GUELLEC \\ 77, rue du Petit-Bois-Tharigné-Fouillard, F 35510 Cesson
}

\begin{abstract}
Résumé
L'objectif de ce travail est de caractériser et de préciser les schémas évolutifs des sols forestiers développés sur la formation de Sologne et sur les sables superficiels issus de son remaniement au quaternaire. Trois grands milieux de pédogenèse ont été proposés : les milieux " secs ", dans lesquels la végétation joue un rôle essentiel dans la différenciation et l'évolution des sols (podzolisation modérée), les zones hydromorphes où se juxtaposent des sols marqués de façon croissante par le lessivage, l'hydromorphie ou la dégradation, les milieux frais, caractérisés par des conditions de station (données biologiques, pédoclimat, matériau) spécifiques.

Les résultats analytiques (critères chimiques et biochimiques) réalisés sur un nombre de profils restreint mais représentatif confirment le diagnostic de la macro-analyse et justifient les coupures biophysiques apportées au paysage solognot.

Les associations forestières reflètent bien les conditions de milieu qui prévalent dans la région, de sorte que le couple sol-végétation présente un intérêt particulier dans loptique d'une cartographie des stations forestières.
\end{abstract}

Mots clés : Caractérisation des sols, relations sol-végétation en Sologne.

\section{Introduction}

Les études globales sur la nature et la genèse des sols développés sur sables, en climat tempéré, ne peuvent se référer qu'à un nombre relativement limité de travaux scientifiques d'ensemble (Folks \& Riecken, 1956 ; Wuerman et al., 1959 ; A meryckx, 1960 ; Kundler, 1961 ; De Coninck \& Laruelle, 1964 ; Juste, 1965 ; Coen \& Pawluk, 1966 ; De Coninck \& Herbillon, 1969 ; Buol, 1973 ; Robin, 1979 ; HerBAUTS, 1981 et 1982). La Sologne, région de grand intérêt écologique, a encore reçu peu d'attention, si l'on excepte le travail réalisé par le C.E.P.E. de Montpellier (Godron, 1964). Des recherches limitées, parfois même ponctuelles, ont cependant été menées et, dans certains secteurs de Sologne ou des zones limitrophes, des types pédogénétiques ont été décrits ou analysés (Duchaufour, 1948 ; Horemans, 1961 ; Ricardo, 1971 ; Aubert, 1974 ; Lelong \& Pich, 1978 ; Guellec, 1980) et des portions de l'espace récemment cartographiés (STuder \& Servant, 1981; Studer, 1983 ; Dupont \& Servant, 1983 ; Racineux, 1983). 
Les profils inventoriés, situés dans une région écoclimatique relativement homogène, ne présentent, au premier abord, rien d'exceptionnel, mais la grande richesse en faciès géologiques et surtout leur grande variabilité géographique posent un problème spécifique d'analyse du milieu. L'objectif de cette étude est de caractériser les sols majeurs formés sur les matériaux sédimentaires meubles qui constituent la formation de Sologne et les sables remaniés de surface et de préciser les schémas évolutifs des profils et leur distribution dans le paysage.

\section{Le cadre géographique}

Le domaine d'étude est centré sur la Sologne des étangs, plus particulièrement sur les communes de Nouan-le-Fuzelier et Salbris.

Les formes du relief sont simples et extrêmement ouvertes. Dans l'ensemble il n'y a pas d'opposition fondamentale plateau-vallée. Les versants rectilignes bien développés sont rares, de même que les plateaux réguliers.

Le secteur de Salbris est essentiellement formé, au Nord de la Sauldre, par un plateau et un glacis légèrement incliné vers le Sud-Ouest. La monotonie de ce plan, où s'emboîtent une série de terrasses, est rompue par la banquette sableuse de La Saulot qui domine le Méant. Au Sud, le domaine des molles ondulations de sables altère l'uniformité des terrasses. Le relief est faiblement échancré par les ruisseaux du Coussin et du Naon. L'altitude ne varie que de 100 à $118 \mathrm{~m}$.

La zone de Nouan a un relief plus indécis. On a affaire à une sorte d'imbrication de paysages de faible étendue, d'échelle réduite. Le relief est plus morcelé, plus serré, plus varié et représente le modelé type de la Sologne des étangs. Le Nant et le Chalès entaillent la couverture de surface mais les différences de niveau sont faibles $(<15 \mathrm{~m})$.

\section{Les méthodes analytiques}

\subsection{Méthodes appliquées aux matériaux parentaux}

- Analyse mécanique de quelques faciès sédimentaires caractéristiques par tamisage sous l'eau (fractions $0-5 \mathrm{~mm}$ ) ; mique.

- Analyse totale par dosage des éléments par spectrométrie d'absorption ato-

\subsection{Méthodes réalisées sur les échantillons de sol (fraction $0-2 \mathrm{~mm}$ )}

- Granulométrie par destruction de la matière organique par l'hypochlorite de sodium, mise en suspension des argiles par action des résines $\mathrm{Na}^{+}$échangeuses d'ions (BURTIN et al., 1972) et prélèvement à la pipette Robinson des différentes fractions.

- Forme des éléments métalliques 
- Détermination des éléments amorphes (Feo, Alo, Mno).

Les portions amorphes des éléments métalliques correspondent aux quantités exprimées en p. 1000 du métal extraites par le réactif de Tamm (oxalate dammonium + acide oxalique $\mathrm{pH} 3,2$ ).

- Détermination des éléments fibres (Fed, Ald. Mnd).

On utilise le réactif MrHRA-JACKSON (1960) qui combine un agent réducteur (dithionite de sodium) et un agent complexant (citrate de sodium) tamponné à pll 7.5 par le bicarbonate de sodium.

- Minéralogie : des diffractogrammes - $X$ de la fraction fine ont été réalisés sur les horizons $\mathrm{A}$ et $\mathrm{B}$ de trois profils caractéristiques des milieux de pédogenèse distingués.

- Etude chimique du complexe absorbant.

Le $\mathrm{pH} \mathrm{H}_{2} \mathrm{O}$ a été mesuré par méthode électrométrique sur un ensemble sol/ solution dans le rapport 1/2.5. Les bases échangeables ont été déterminées selon la méthode classique par percolation à lacétate d'ammonium tamponné à $\mathrm{pH} 7$. Les cations acides ont été extraits par le chlorure de potassium normal et dosés par un titrimètre automatique (Tacussel Urectron 5) jusqu'à un $\mathrm{pH}$ limite de 8,4 .

La sommation des bases échangeables évaluées à pH 7 et des acidités donne la capacité totale d'échange au $\mathrm{pH}$ du sol (Te).

- Fractionnement de la matière organique et analyse des complexes organominéraux.

- Etude des humus.

L'étude des substances organiques s'effectue par deux types d'opérations:

- un fractionnement physique par tamisage sous l'eau des échantillons sur tamis de 100 , puis $50 \mu \mathrm{m}$. La fraction $2000-100 \mu \mathrm{m}$ est recueillie sur filtre pesé, la fraction 0 $50 \mu \mathrm{m}$ obtenue par centrifugation et séchage à $45^{\circ} \mathrm{C}$. Le rapport de ces deux phases précise l'état de division et d'intégration de la matière organique. Par ailleurs, une observation de lhorizon $A_{1}$ à la loupe binoculaire en lumière naturelle a été systématiquement réalisée ;

- un fractionnement chimique appliqué sur la phase fine $(<50 \mu \mathrm{m})$, visant à séparer les composés organiques du sol en fonction du mode de liaison organo-minéral qui les caractérise. Les réactifs d'extraction employés successivement sont le tétraborate

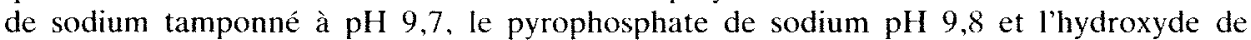
sodium $\mathrm{pH}$ 12. Deux extractions, respectivement par agitation de 1 et $1 / 2$ heure, sont effectuées en utilisant des rapports $1 \mathrm{~g}$ de sol/100 $\mathrm{ml}$ de solution.

L'adjonction d'un acide (HCL $2 \mathrm{~N}$ ) aux solutions d'extraction permet de séparer les acides fulviques (AF) qui restent solubles et les acides humiques (AH) qui précipitent en petits flocons à $\mathrm{pH} 1,5$.

- Etude des complexes organo-minéraux

Des extractions par le tétraborate et le pyrophosphate de sodium ont été opérées sur les horizons $A_{1}$ et les horizons de transition ou diagnostiques (sol global). L'action spécifique des deux réactifs apparaît dans les quantités de fer et d'aluminium libérées par les deux traitements. 


\section{Le milieu biophysique}

\subsection{L'environnement géologique}

Le territoire solognot est formé de terrains détritiques tertiaires essentiellement burdigaliens et helvétiens (Gigout et al., 1972; RasPlus, 1978) qui remplissent une vaste cuvette tectonique formée pendant l'Eocène et l'Oligocène jusqu'à l'Aquitanien.

La formation de Sologne constitue un vaste ensemble globalement uniforme où alternent de manière discontinue horizontalement et verticalement de nombreux faciès sédimentaires (argile, argile sableuse, sable argileux, sable moyen et grossier plus ou moins pourvu en éléments fins...) dont le grain moyen varie largement (de 0,18 à $0,8 \mathrm{~mm}$ pour les matériaux analysés dans cette étude). Les sédiments s'organisent en faisceaux argileux ou sableux subhorizontaux ou, le plus souvent inclinés (de 0 à 40 p. 100). La structure du corps sédimentaire se présente en lentilles de sables et d'argile qu'il n'est respectivement pas possible de corréler d'un point à un autre. En fait, la granulométrie des faciès peut varier brutalement sur de courtes distances. Ainsi, la fraction $0-50 \mu \mathrm{m}$, qui constitue un peu moins de $10 \mathrm{p}$. 100 des volumes réduits et de l'ordre de 19 p. 100 dans les domaines oxydés du Burdigalien grossier (échantillon global), s'élève à plus de $30 \mathrm{p} .100$ dans les stations (1) éloignées parfois de quelques dizaines de mètres et jusqu'à 74 p. 100 dans le Burdigalien argilo-limoneux prospecté dans la même zone.

Un peu partout sur le territoire solognot, on constate la présence, au-dessus des sables brun orange ou jaune orange rubéfiés du sommet de la formation de Sologne, de sables moyens ou grossiers gris beige à beige, feldspathiques, accumulés sur 1 ou $2 \mathrm{~m}$ d'épaisseur, rarement plus. Cette couverture, qui masque plus ou moins complètement les sables et argiles du corps sédimentaire solognot, occupe de grandes surfaces, en particulier à Nouan-le-Fuzelier. Sa caractéristique essentielle est de montrer un mélange intime de toutes les fractions granulométriques (LE Houerou, 1961) ce qui est la marque d'un brassage important par les agents périglaciaires. Ces sables remaniés («sables morts»), de consistance friable, sont essentiellement des sables moyens et grossiers (chaque fraction représente en général un peu plus de 30 p. 100 de l'ensemble de l'échantillon). Les sables très grossiers sont nettement minoritaires $(10 \mathrm{p} .100) \mathrm{de}$ même que les sables fins et très fins. La teneur vraiment très faible en limons et en argile (L'ensemble de ces deux fractions avoisinent 10 p. 100), la quasi-absence de graviers et de cailloux sont les autres caractères majeurs de ces faciès remaniés.

Ces matériaux se caractérisent par la grande abondance de quartz et par la présence de quantités non négligeables de feldspaths potassiques (orthose, microcline) et les analyses chimiques (cf. tabl. 1) révèlent une roche très acide (on note toujours plus de 70 p. 100 de silice), extrêmement pauvre en bases, bien pourvue en potassium et surtout en aluminium. Les pourcentages de fer tombent à des valeurs très basses dans les sables remaniés. Par ailleurs, ces faciès renferment des micro-éléments et des éléments traces en quantités variables. Des carences existent pour le bore, le molybdène, le cuivre et le manganèse. Les proportions de zirconium, d'acide phosphorique et surtout de baryum sont au contraire relativement importantes (GuELLeC, 1982).

(1) Le type de sédiment rencontré dans ces stations sensiblement plus riche en argile et en limons que le Burdigalien grossier semble, dans certaines conditions hydrodynamiques, favoriser les processus de planosolisation. 
Au total, l'existence de nombreux faciès sédimentaires aux caractères intrinsèques différentiels (granulométrie, teneur en minéraux facilement altérables, densité, couleur, cortège minéralogique) et leur grande variabilité dans un cadre spatial limité d'une part, la paléopédogenèse et le travail de l'érosion différentielle qui se sont exercés durant le quaternaire, d'autre part, contribuent à multiplier les modèles morphopédologiques (dôme sableux, banquette et bourrelet alluviaux, glacis, croupe, terrasse, versants plus ou moins réguliers, dépressions ouvertes, placage sableux plus ou moins bien conservé, colluvions...). La richesse en nuances édaphiques démontre l'intérêt de pouvoir calibrer les matériaux répertoriés et de préciser leur zonéographie dans le district écologique considéré.

TABLEAU 1

Analyse totale de quelques sédiments solognots (en p. 100).

Total analysis of some parent materials of sologne.

\begin{tabular}{c|c|c|c|c|c|c|c|c}
\hline \multicolumn{1}{c|}{ Elément } \\
$\begin{array}{l}\text { Matériau } \\
\text { grain moyen) }\end{array}$ & $\mathrm{S}_{\mathrm{i}} \mathrm{O}_{2}$ & $\mathrm{Al}_{2} \mathrm{O}_{3}$ & $\mathrm{Fe}_{2} \mathrm{O}_{3}$ & $\mathrm{CaO}$ & $\mathrm{MgO}$ & $\mathrm{K}_{2} \mathrm{O}$ & $\mathrm{MnO}$ & $\mathrm{T}_{i} \mathrm{O}_{2}$ \\
\hline $1(0,35)$ & 91 & 4,9 & 0,4 & 0,1 & 0,1 & 3,2 & 0,01 & 0,24 \\
$2(0,32)$ & 88,5 & 6,7 & 1 & 0,1 & 0,1 & 3,3 & 0,01 & 0,30 \\
$3(0,80)$ & 86,5 & 7,3 & 2,6 & 0,1 & 0,1 & 3,2 & 0,01 & 0,13 \\
$4(0,75)$ & 89,8 & 6,2 & 0,5 & 0,1 & 0,1 & 3,2 & 0,01 & 0,10 \\
$5(0,18)$ & 74,2 & 16 & 5,4 & 0,2 & 0,6 & 2,7 & 0,01 & 0,91 \\
$6(0,40)$ & 85,8 & 8,3 & 2,1 & 0,1 & 0,2 & 3,1 & 0,01 & 0,37 \\
\hline
\end{tabular}

1: sabies remaniés: 2 : palésol : $3-4$ : burdigalien grossicr oxydè ct réduit de la même formation (faciès sableux) ; 5 : burdigalien (faciès argilo-limoneux) ; 6 : sable argileux masquant le burdigalien grossier.

\subsection{Les caractéristiques climatiques}

Le régime macroclimatique, de type océanique dégradé, est sensiblement perturbé par les facteurs stationnels (matériau, topographie, végétation). Les données pédoclimatiques opposent significativement trois grands milieux biophysiques: milieux frais, milieux secs et zones hydromorphes.

Dans les zones hydromorphes, froides et lentes à se réchauffer, le rôle « hydrogénétique * de la nappe apparaît fondamental et les variations de l'économie en eau (durée d'engorgement, présence ou non d'une période sèche, contraste saisonnier) sont un facteur de différenciation pédologique majeur.

Dans les milieux « secs », le fait essentiel est l'existence d'une période de dessiccation prononcée des profils et, plus particulièrement, des horizons de surface, qui est favorisée (lande boisée ou bétulaie ouverte à éricacées) ou, au contraire, quelque peu atténuée (formations à mousses ou à lichens, futaies de résineux) par la végétation. Le pouvoir évaporant de l’air y est très élevé en été et les amplitudes diurnes de la température du sol importantes. C'est surtout le cas à Salbris où peuvent exister des périodes " sub-sèches" de l'ordre de 60 jours (GAUSSEN, 1955). 
Les sols sains ou légèrement affectés par l'hydromorphie qui se développent le long des versants ou dans les parties basses de la topographie, reçoivent, en quelque sorte, un surplus de précipitations par ruissellement ou écoulement latéral et conservent une réserve en eau satisfaisante tout au long de l'année d'autant que ces biotopes sont généralement ombragés (formations de feuillus plus ou moins fermées) et mieux abrités. Les milieux frais, de caractère intermédiaire, qui se forment dans une couverture pédologique relativement limitée $(\leqslant 1 \mathrm{~m})$, ne connaissent pas les contrastes microclimatiques brutaux qui caractérisent les zones hydromorphes et les milieux " secs » au cours de l’année.

\subsection{Le rôle de la végétation}

La forêt de feuillus, plus précisément la chênaie acidiphile (Quercion occidentale) qui représente probablement la végétation climacique de la Sologne (BrAun-BLanquet, 1967) a été profondément modifiée et. en grande partie, détruite par l'homme.

La chênaie acidiphile typique n'existe effectivement qu'à l'état de lambeaux et le Chêne (Quercus robur, Quercus petraea, Quercus pyrenaica) ne forme guère plus que des petits massifs ou des franges étroites s'échelonnant le long des vallons et vallées. Le plus souvent, on rencontre des formations plus ou moins dégradées : chênaie claire, chênaie à canche, chênaie à fougère-aigle, à Ericacées, à molinie, ou à un stade plus avancé la chênaie-bétulaie.

Le Bouleau (Betula verrucosa, Betula pubescens) est un très bon pionnier qui a beaucoup colonisé les domaines trop secs ou trop humides en évolution. Plusieurs types sont à signaler : variantes à Ericacées, à lichens (milieux «secs»), à ronces et graminées, à mousses, à molinie (zones hydromorphes), à fougère-aigle, à graminées (milieux frais). Cette essence forestière se présente généralement en association avec le chêne, le pin, le châtaignier et, dans les faciès plus humides, le saule, le tremble, l'aulne, essentiellement sous forme de taillis sous futaie ou de bosquets (bétulaies à lichens...) mais aussi de futaies de belle venue (certaines bétulaies à fougère-aigle).

Les résineux (essentiellement Pinus laricio, Pinus sylvestris, et aussi Pinus strobus, Pinus pinaster, Pseudotsuga Douglasii, Abies alba, Abies grandis, Abies Nordmanannia...) couvrent de grandes surfaces et dominent de plus en plus les futaies de Sologne.

Cependant, le trait le plus frappant du paysage solognot est la présence de landes à Ericacées (Calluna vulgaris, Erica cinerea), en régression, et surtout de landes boisées émaillées de bouleaux, de pins et de chênes.

\section{Les profils d'études}

Les profils les plus caractéristiques de chaque milieu de pédogenèse ont été sélectionnés en vue d'un diagnostic approfondi. Ce sont deux sols bruns ocreux (milieux " secs »), un sol brun mésotrophe et un sol brun acide affecté par des processus encore discrets de lessivage et de marmorisation (milieux frais), un sol lessivé hydromorphe planosolique et un sol lessivé hydromorphe podzolique (zones hydromorphes). 
Les séquences pédogénétiques que l'on enregistre en Sologne et les groupements végétaux qui leur sont associés déterminent, avec les conditions physiographiques, un paysage caractéristique.

\section{Caractérisation de la couverture pédologique. Critères de différenciation}

\subsection{Critères texturaux}

Un caractère spécifique des profils développés en milieu hydromorphe est la présence en profondeur d'un horizon argillique ou d'un substrat relativement imperméable (lit argileux ou sablo-argileux. terrasse, paléosol). Le matériau, la configuration du toit géologique et la topographie conjuguent leurs effets pour créer des modèles pédogénétiques plus ou moins typés.

Le sol lessivé hydromorphe se caractérise par des teneurs en limons déjà importantes, de l'ordre de 20 à 24 p. 100, vu le contexte sableux de ces milieux (cf. tabl. 2). Le rapport limon/argile avoisine ou dépasse 3 dans les niveaux supérieurs. Le rapport limon fin/argile se situe entre 1,85 et 2 . Le fait majeur est la discontinuité texturale relativement brutale qui existe entre les horizons A et B: l'indice d'entraînement apparent de l'argile est de l'ordre de 3,9, ce qui traduit un transfert important.

Dans le sol lessivé hydromorphe podzolique, les quantités de sables sont très élevées dans tous les horizons, de l'ordre de 68 p. 100 en Btg (contre un peu moins de 52 p. 100 dans le profil précédent) et de 79 p. 100 en A21 (contre 69 p. 100) et il s'agit surtout de sables grossiers. Le pourcentage de limons se situe autour de 15 à 18 p. 100 . On observe un départ important d'argile des horizons $A$ et une concentration en $B$ : l'indice d'entraînement apparent (Maxi B/Mini A21) est égal à 3,4.

Les différences granulométriques qui existent entre ces deux profils correspondent aussi à des différences de matériau parental, sable argileux dans un cas (matériau 6), sable grossier dans l'autre (matériau 3 et 4 ).

Mécaniquement, Ies milieux "secs" s'opposent totalement aux zones hydromorphes. Les éléments grossiers deviennent largement prédominants : on y dénote plus de 80 p. 100 de sables dans les horizons B et parfois jusqu'à 95 p. 100 en B/C. Le caractère frappant est la pauvreté extrême en argile, en particulier à Nouan-le-Fuzelier où les teneurs en $\mathrm{B}$ avoisinent $2,5 \mathrm{p}$. 100. Il est à noter que le lessivage n'a pas de prise sur ce type de formation ou reste un processus tout à fait secondaire.

Les sables remaniés des milieux frais s'apparentent à ceux des milieux secs, mais dans certaines situations, un processus de colluvionnement a enrichi le matériau en argile (X 1.7 en B dans des exemples étudiés) ou en limons (X 1.6). En outre, la part des sables grossiers est, comparativement aux sables fins, sensiblement moins conséquente.

\subsection{Le complexe absorbant}

Les bases échangeables

La garniture ionique du complexe est fonction des conditions de station (nature du matériau en particulier) et du type de pédogenèse. Les profils affectés par la podzolisa- 


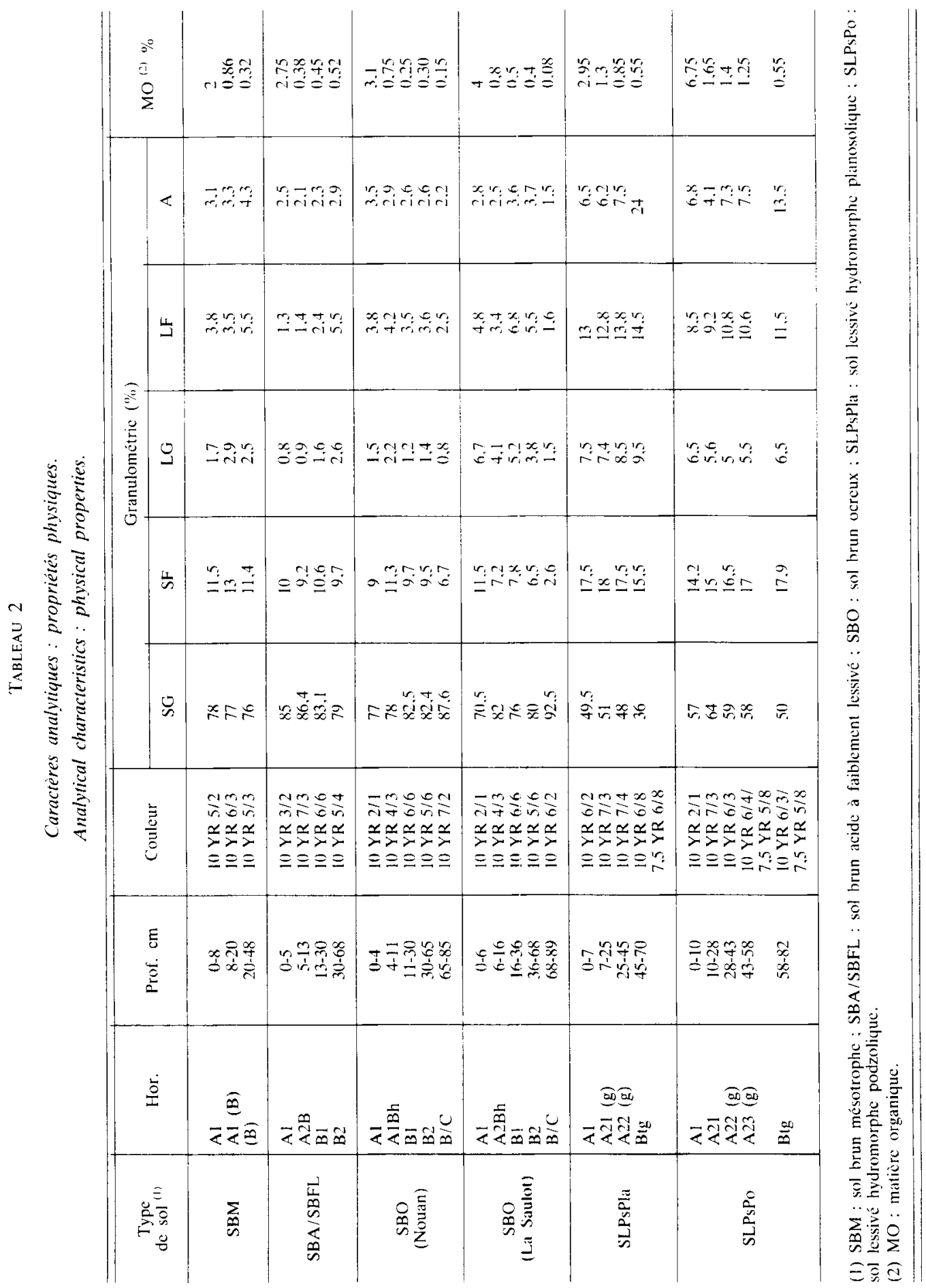


tion ou la dégradation sont les plus désaturés. Le calcium constitue l'élément majeur du complexe mais les rapports $\mathrm{Ca} / \mathrm{Mg}$ et même $\mathrm{Ca} / \mathrm{K}$ sont, dans la plupart des cas, un peu faibles.

Un changement s'opère dans le rapport quantitatif des cations basiques le long des profils. Plus particulièrement, l'évolution du rapport $\mathrm{Ca} / \mathrm{Mg}$ sépare fondamentalement les zones hydromorphes d'une part, les milieux frais et « secs » d'autre part. C'est ainsi que dans les stations hydromorphes se produit un accroissement en valeur relative conséquent de l'ion $\mathrm{Mg}^{2+}$ en profondeur. Ce processus est peu perceptible dans le sol lessivé hydromorphe planosolique où le rapport $\mathrm{Ca} / \mathrm{Mg}$ passe de 4,8 à 3,6 en $\mathrm{Btg}$, mais il devient prépondérant dans le sol lessivé hydromorphe podzolique où ce rapport, égal à 5.5 dans l'horizon de surface, diminue régulièrement le long du profil puis chute en A23g $(2,1)$ et en Btg $(1,9)$, qui sont les niveaux actuellement les plus affectés par la dégradation. Au contraire, le rapport $\mathrm{Ca} / \mathrm{Mg}$ offre une dynamique tout à fait différente en milieu « sec", où on enregistre une augmentation graduelle de ce rapport de haut en bas des profils, et en milieu frais, où les proportions relatives de ces deux éléments restent à peu près identiques le long des profils. La part du magnésium est en valeur relative sensiblement plus forte dans ces stations de bas de versants $(\mathrm{Ca} / \mathrm{Mg} \leqslant 3,5)$.

\section{Les acidités d'échange}

Les cations acides échangeables $\mathrm{Al}^{3+}$ et $\mathrm{H}^{+}$gouvernent, par leur importance, le degré d'insaturation du complexe et l'agressivité des solutions du sol.

Dans les zones hydromorphes, les taux d'acidité d'échange $\left(A_{e} / T_{e}\right)$ sont maxima, et en outre parfois (sols hydromorphes dégradés) très élevés $(>60 \mathrm{p} .100)$, dans les horizons superficiels et diminuent progressivement le long du profil ou brutalement en B.

En milieu sec, les maxima d'acidité se situent dans les horizons minéraux et, plus précisément, en $\mathrm{B} 1$ ou en $\mathrm{A} 1 \mathrm{Bh}$ où les taux dépassent le plus souvent $60 \mathrm{p} .100$. Un minimum peu accusé apparaît dans l'horizon de surface ou en B/C.

Dans les milieux frais, l'acidité d'échange, qui reste moyennement élevée $(<50$ p. 100 ), augmente franchement de haut en bas des profils. Un maximum bien marqué se situe dans les horizons B.

L'aluminium ( $\mathrm{A} 1^{3+}$, polymères hydroxy- $\mathrm{Al}$ ) est le cation acide prédominant dans toutes les stations, mais le potentiel protonique différencie bien le type de pédogenèse en milieu sain : les protons échangeables, qui sont peu représentés dans les sols brunifiés à humus biologiquement actif de type mull ou mull-moder $\left(\mathrm{H}^{+} \# 5\right.$ p. 100), deviennent très abondants dans les sols brunifiés podzoliques et les rapports $\mathrm{H}^{+} / \mathrm{Al}^{3+}$, qui varient de 0,62 (Salbris) à 0,85 (Nouan) en $\mathrm{A} 1$, rendent compte de l'agressivité des acides organiques.

\subsection{Les oxydes}

L'analyse des formes du fer et de l'aluminium et leur distribution verticale en valeur absolue et par rapport à l'argile permettent de préciser le type et le degré de l'altération pédochimique dans les différentes zones échantillonnées. 


\begin{tabular}{|c|c|c|c|c|c|c|c|c|}
\hline & $\sum_{0}^{\infty \pi}$ & $\begin{array}{l}\text { nan } \\
\text { ninim }\end{array}$ & $\begin{array}{l}\text { con } \\
\text { cinicin }\end{array}$ & miner & $n_{i}+r_{i} N$ & 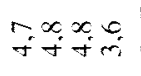 & 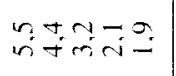 & \\
\hline & $\stackrel{*}{*}$ & $\nexists \bar{\infty} \overline{i n}$ & EFm & 복요 & $\ddot{B} \approx \simeq m \tilde{m}$ & トマミッ & 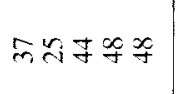 & \\
\hline & 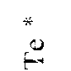 & 공 & $\overrightarrow{3}=0$ & 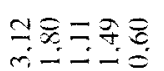 & 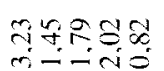 & 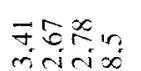 & $\begin{array}{l}x \\
\dot{y} \\
\dot{y}\end{array}=\frac{n}{q}$ & \\
\hline & $\stackrel{\stackrel{\omega}{2}}{*}$ & 메 & 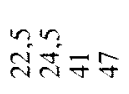 & $\stackrel{n}{\infty} \underset{\infty}{\infty}$ & 于 858 与 & mnd & 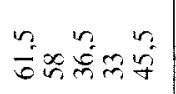 & \pm \\
\hline$\Sigma$ & t & $\begin{array}{l}9 \\
0\end{array}$ & 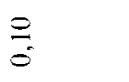 & 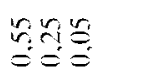 & 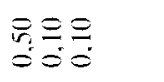 & $\stackrel{9}{90}$ & $\begin{array}{l}n= \\
\stackrel{0}{0}=\end{array}$ & $\begin{array}{l}\Xi \\
\Xi\end{array}$ \\
\hline$\stackrel{z}{\approx}$ & $\frac{m}{2}$ & $\begin{array}{l}0.00 \\
0: 00\end{array}$ & 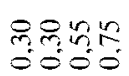 & 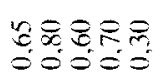 & 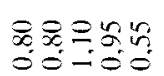 & 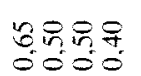 & 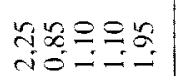 & בั \\
\hline $\begin{array}{l}5 \\
8 \\
8\end{array}$ & $n$ & $\begin{array}{l}\infty{ }_{0}^{\infty} \infty \\
-0=\end{array}$ & 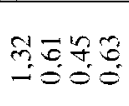 & 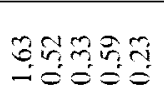 & త્વે & 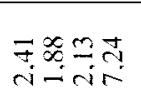 & 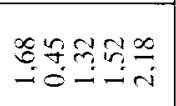 & $\$$ \\
\hline $\begin{array}{l}\bar{\Xi} \\
\bar{\Xi} \\
\bar{\Xi}\end{array}$ & $\Sigma$ & $\begin{array}{l}085 \\
085 \\
=0\end{array}$ & $\begin{array}{l}0.00 \\
0.00 \\
0\end{array}$ & $\stackrel{+}{0}$ & & $\begin{array}{l}599 \\
090 \\
0\end{array}$ & & 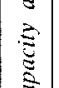 \\
\hline$\frac{\text { a }}{3}$ & $\ddot{z}$ & 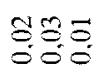 & 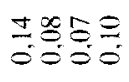 & 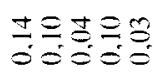 & 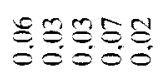 & $\begin{array}{l}\text { บㅗ응 } \\
0000\end{array}$ & 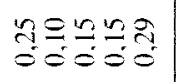 & (ֶ) \\
\hline 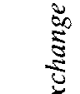 & 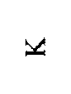 & $\begin{array}{l}2 \pm= \\
6=0\end{array}$ & लি: & 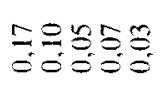 & $\begin{array}{l}0.50 \\
0\end{array}$ & $\Xi=\frac{1}{0}=0$ & $\frac{2}{0}: \frac{9}{0}=\frac{1}{0}$ & \\
\hline $\begin{array}{l}\dddot{3} \\
3\end{array}$ & $\sum^{\infty}$ & 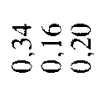 & 궁응ㅇㅇㅇ & 동영영 & 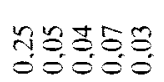 & ๑ேণ & 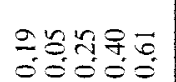 & $\underline{z}$ \\
\hline 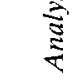 & 8 & 원영 & 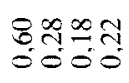 & 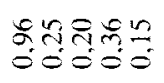 & 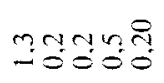 & 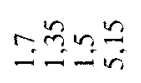 & 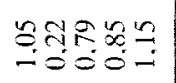 & 吾 \\
\hline & $\begin{array}{l}\stackrel{\mathbb{I}}{I} \\
\underline{I}\end{array}$ & yó & 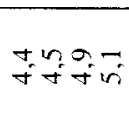 & 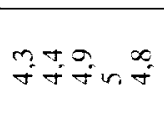 & 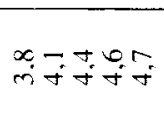 & neviva & 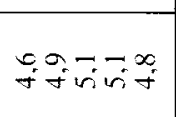 & 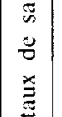 \\
\hline & $\dot{5}$ & 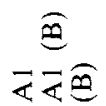 & 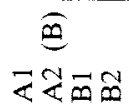 & 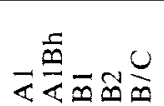 & 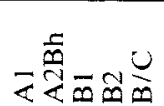 & 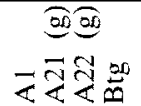 & 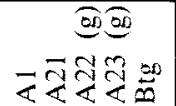 & 吾 \\
\hline & $\begin{array}{l}2 \overline{8} \\
\sum^{2}\end{array}$ & $\sum_{\infty}^{\infty}$ & 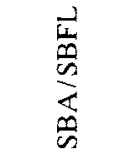 & 危 & 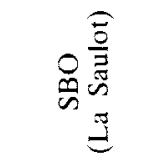 & $\frac{\frac{\pi}{2}}{\frac{\pi}{n}}$ & $\begin{array}{l}0 \\
\frac{1}{n} \\
\frac{1}{n}\end{array}$ & 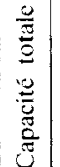 \\
\hline
\end{tabular}




\section{Les zones hydromorphes}

L'ambiance physico-chimique qui prévaut dans ce milieu et l'action de la matière organique déclenchent un certain nombre de mécanismes (changement d'état physique, sursaturation hydrique, mobilisation et redistribution des éléments métalliques au sein du profil, chéluviation, élimination des bases...) qui conduisent à un résultat d'expression morphologique plus ou moins comparable.

Dans les profils types inventoriés, le rapport Fed/argile augmente brutalement en Btg (cf. tabl. 4). Les maxima et les minima de ce rapport sont aussi les maxima et minima de fer libre, ce qui tend à démontrer la migration séparée des deux éléments. le fer précédant l'argile. Les indices d'entraînement du fer sont effectivement nettement supérieurs à ceux de l'argile (cf. fig. 1). Ces indices, qui semblent énormes (16 pour le sol lessivé hydromorphe planosolique, 21 pour le sol lessivé hydromorphe podzolique), laissent supposer l'existence d'un apport latéral dans les horizons de profondeur ou bien d'un processus d'appauvrissement dans les niveaux de surface, qui ajouteraient leurs effets au lessivage ou à la dégradation.

TABI.EAU 4

Distribution des orydes de fer et d'aluminium le long des profils.

Distribution of iron and aluminium oxydes down the profiles.

\begin{tabular}{|c|c|c|c|c|c|c|c|c|}
\hline $\begin{array}{l}\text { Type } \\
\text { de sol }\end{array}$ & Hor. & $\begin{array}{l}\text { Fed } \\
(\%)\end{array}$ & $\begin{array}{l}\text { Ald } \\
(\% \circ)\end{array}$ & $\mathrm{Fed} / \mathrm{a}$ & Ald $/ \mathrm{a}$ & Feo/Fed & Alo/Ald & Feo/Alo \\
\hline SBM & $\begin{array}{l}\text { A1 } \\
\text { AlB } \\
\text { (B) }\end{array}$ & $\begin{array}{l}0,75 \\
0,75 \\
1\end{array}$ & $\begin{array}{l}0.3 \\
0.5 \\
0.5\end{array}$ & $\begin{array}{l}2,4 \\
2,3 \\
2,3\end{array}$ & $\begin{array}{l}1 \\
1,5 \\
1.2\end{array}$ & $\begin{array}{l}0,27 \\
0,4 \\
0,45\end{array}$ & $\begin{array}{l}0,4 \\
0,5 \\
1\end{array}$ & $\begin{array}{l}1,65 \\
1,2 \\
0,9\end{array}$ \\
\hline $\mathrm{SBA} / \mathrm{SBF}$ & $\begin{array}{l}\text { A1 } \\
\text { A2B } \\
\text { B1 } \\
\text { B2 }\end{array}$ & $\begin{array}{l}0,3 \\
0,25 \\
0,5 \\
0.7\end{array}$ & $\begin{array}{l}0,15 \\
0,15 \\
0,25 \\
0,3\end{array}$ & $\begin{array}{l}1,2 \\
1,2 \\
2,2 \\
2,4\end{array}$ & $\begin{array}{l}0,6 \\
0,7 \\
1 \\
1 \\
\end{array}$ & $\begin{array}{l}0,33 \\
0,4 \\
0,5 \\
0,85\end{array}$ & $\begin{array}{l}0,35 \\
0,35 \\
0,4 \\
1\end{array}$ & $\begin{array}{l}2 \\
2 \\
2,5 \\
2\end{array}$ \\
\hline $\begin{array}{c}\text { SBO } \\
\text { (Nouan) }\end{array}$ & $\begin{array}{l}\mathrm{A} 1 \\
\mathrm{~A} 1 \mathrm{Bh} \\
\mathrm{B} 1 \\
\mathrm{~B} 2 \\
\mathrm{~B} / \mathrm{C}\end{array}$ & $\begin{array}{l}0.6 \\
0.6 \\
0.8 \\
0,8 \\
0.5\end{array}$ & $\begin{array}{l}0.2 \\
0.2 \\
0.5 \\
0.8 \\
0.4\end{array}$ & $\begin{array}{l}1,7 \\
2,1 \\
3,1 \\
3,1 \\
2,3\end{array}$ & $\begin{array}{l}0.57 \\
0.69 \\
1.9 \\
3 \\
1.8\end{array}$ & $\begin{array}{l}0,33 \\
0,67 \\
0,75 \\
0,62 \\
0,40\end{array}$ & $\begin{array}{l}0,5 \\
0,5 \\
0,8 \\
0,75 \\
1\end{array}$ & $\begin{array}{l}2,5 \\
3,5 \\
2 \\
2,1 \\
1,65\end{array}$ \\
\hline $\begin{array}{c}\text { SBO } \\
\text { (La Saulot) }\end{array}$ & $\begin{array}{l}\mathrm{A} 1 \\
\mathrm{~A} 2 \mathrm{Bh} \\
\mathrm{B} 1 \\
\mathrm{~B} 2 \\
\mathrm{~B} / \mathrm{C}\end{array}$ & $\begin{array}{l}0.7 \\
1 \\
1.75 \\
1.75 \\
0.5\end{array}$ & $\begin{array}{l}0.15 \\
0.15 \\
0.5 \\
0.5 \\
0.12\end{array}$ & $\begin{array}{l}2,5 \\
4 \\
4.85 \\
4,7 \\
3,3\end{array}$ & $\begin{array}{l}0.54 \\
0.6 \\
1,38 \\
1,35 \\
0.8\end{array}$ & $\begin{array}{l}0,35 \\
0,35 \\
0,52 \\
0,48 \\
0,40\end{array}$ & $\begin{array}{l}0,67 \\
0,67 \\
0,9 \\
0,8 \\
1\end{array}$ & $\begin{array}{l}2 \\
4 \\
1,5 \\
0,83 \\
0,5\end{array}$ \\
\hline SLPsPla & $\begin{array}{l}\text { A1 } \\
\text { A21 (g) } \\
\text { A22 (g) } \\
\text { Btg }\end{array}$ & $\begin{array}{r}0,75 \\
0,75 \\
1,25 \\
12 \\
\end{array}$ & $\begin{array}{l}0.25 \\
0.25 \\
0.5 \\
1.5 \\
\end{array}$ & $\begin{array}{l}1,15 \\
1,2 \\
1,65 \\
5 \\
\end{array}$ & $\begin{array}{l}0.38 \\
0.4 \\
0,66 \\
0,62 \\
\end{array}$ & $\begin{array}{l}0,73 \\
0,60 \\
0,44 \\
0,33\end{array}$ & $\begin{array}{l}1 \\
1 \\
0,4 \\
0.4\end{array}$ & $\begin{array}{l}2,2 \\
1,8 \\
2,75 \\
6,6 \\
\end{array}$ \\
\hline SLPsPo & $\begin{array}{l}\text { A1 } \\
\text { A21 } \\
\text { A22 }(\mathrm{g}) \\
\text { A23 }(\mathrm{g}) \\
\text { Btg }\end{array}$ & $\begin{array}{l}0,75 \\
0,25 \\
1 \\
1 \\
5,25\end{array}$ & $\begin{array}{l}1 \\
0,6 \\
0.7 \\
0.75 \\
1\end{array}$ & $\begin{array}{l}1,1 \\
0,6 \\
1,37 \\
1,33 \\
3,9\end{array}$ & $\begin{array}{l}1.5 \\
1,5 \\
0,96 \\
1 \\
0,75\end{array}$ & $\begin{array}{l}0,66 \\
0,40 \\
0,65 \\
0,65 \\
0,35\end{array}$ & $\begin{array}{l}0,9 \\
0,9 \\
1 \\
1 \\
0,65\end{array}$ & $\begin{array}{l}0,55 \\
0,18 \\
0,93 \\
0,85 \\
2,85\end{array}$ \\
\hline
\end{tabular}


SBM
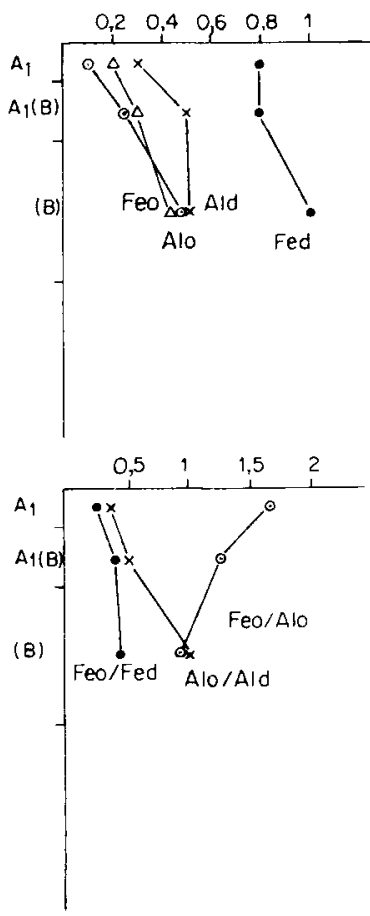

SBO
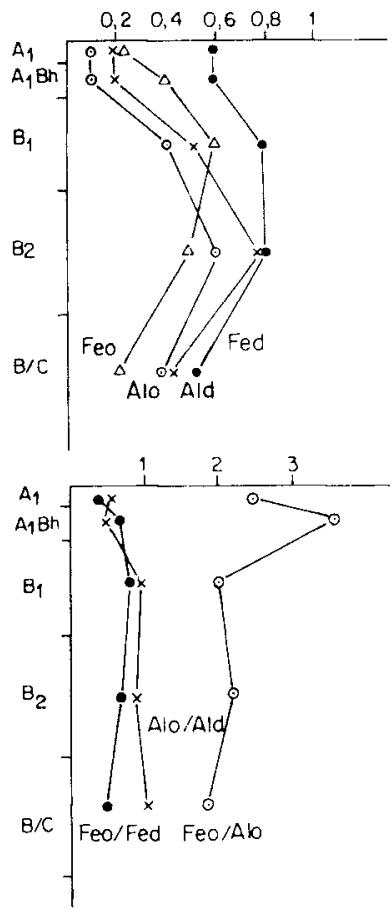
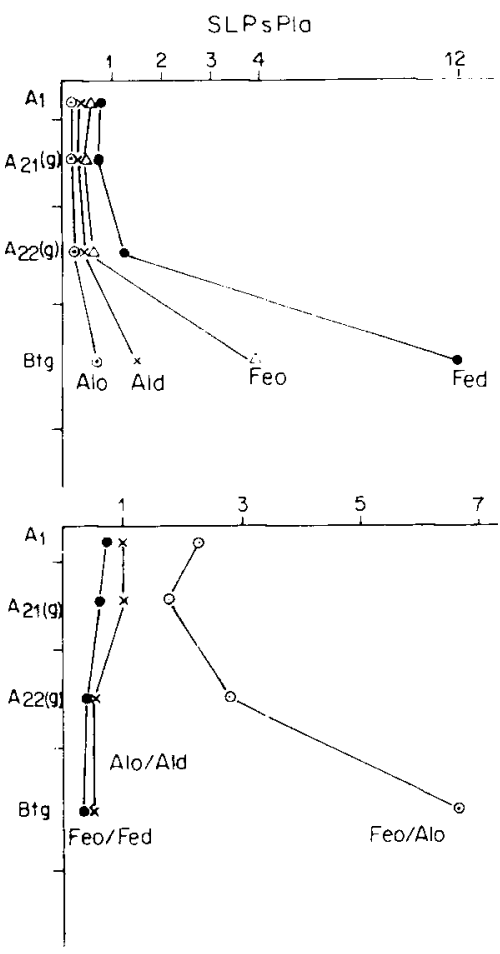
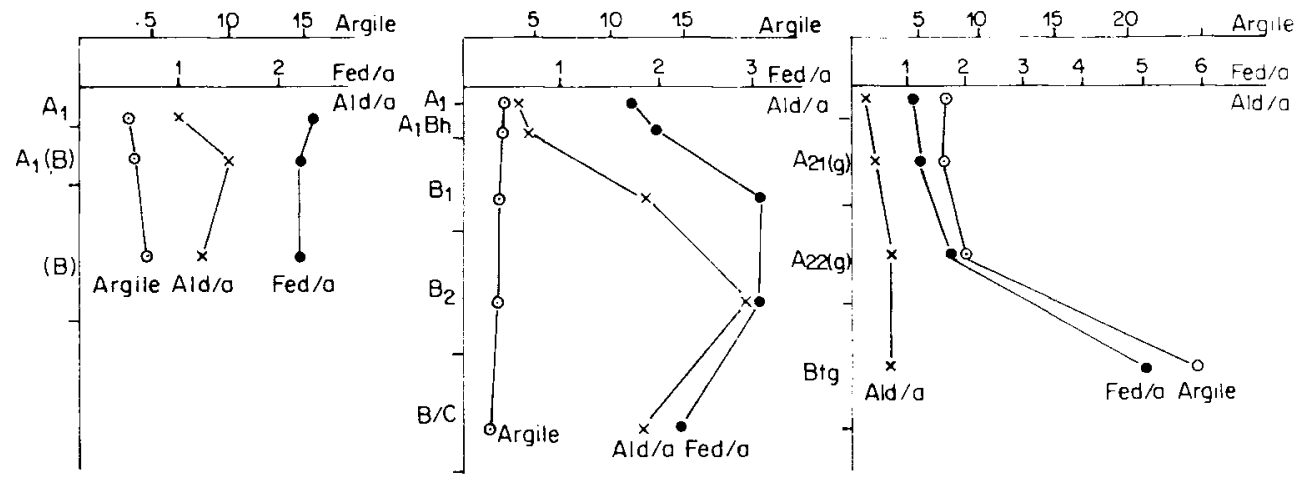

Figi. 1

Distribution des oxydes de fer et daluminium at de largile dans les 3 profils caractéristiques. Depth functions of iron and aluminitum orydes and clay of three characteristic profiles.

a : argile - clay.

0 : formes amorphes - amorphous portion.

d : formes libres - free eloments. 
Un autre trait caractéristique dans ces sols est la diminution plus ou moins progressive des rapports FeolFed de la surface vers la base des profils. Ce fait oppose totalement ces profils aux sols non influencés par une nappe qui se développe dans les sables remaniés. Les valeurs rencontrées dans les horizons superficiels $(\geqslant 0,5)$ traduisent l'abondance des formes amorphes. L'hydromorphie semi-permanente qui caractérise ces sols et la richesse en carbone (on note encore 1,25 p. 100 de matière organique à plus de $50 \mathrm{~cm}$ dans le sol lessivé hydromorphe podzolique) retardent ou empêchent le vieillissement des oxydes de fer (Blume \& Schwertmann, 1969). Les chiffres observés dans les horizons illuviaux $(\leqslant 0,35)$ signifient que les oxydes et hydroxydes de fer cristallisés prédominent nettement. Ce fait est probablement, pour une part, à mettre en relation avec un phénomène d'illuviation d'argile et de fer évolué, cristallin étroitement associés, antérieure à l'installation de la nappe dans la tranche supérieure du profil et à l'accentuation du lessivage.

L'aluminium ne s'accumule pas comme le fer, ce qui est dans l'ordre des choses. Le rapport Ald/argile, qui peut constituer un indice d'altération des argiles, reste très bas et du mème ordre de grandeur de haut en bas des profils. Le rapport Alo/Ald semble traduire, mais de manière beaucoup moins exprimée, un comportement de l'aluminium comparable à celui du fer.

\section{Les milieux "secs"}

Le caractère dominant des sols forestiers installés sur interfluves sableux est l'augmentation parfois sensible des rapports Feo/Fed et Alo/Ald des horizons de surface vers la profondeur. C"est plus net pour l'intergrade podzolique (sol brun ocreux) de Nouan-le-Fuzelier, où un maximum relatif bien marqué apparaît pour Feo/Fed en B1 et B2, que pour le profil observé sur la «banquette" alluviale de Salbris où un processus de lessivage modéré a précédé la podzolisation ménagée qui oriente actuellement l'évolution de ce profil.

Les rapports Fed/argile et Ald/argile présentent une fonction analogue qui montre un maximum relativement bien perceptible dans les horizons $B$.

Cette double information traduit une redistribution du fer et de laluminium ménagée au sein des profils et témoigne d'une podzolisation commençante dans ces sols.

Ce type d'évolution est également mis en évidence par le rapport Feo/Alo qui présente un maximum marqué dans les horizons diagnostiques A1 Bh et A2 Bh.

\section{Les milieux frais}

Dans le sol brun mésotrophe, le fer libéré par l'altération a tendance à rester sur place, dans sa zone de formation, et à s'associer avec l'argile. L'indice d'entraînement que l'on enregistre $\left(I_{\mathrm{fe}}=1,3\right)$, vraiment très faible s'explique probablement par une légère dispersion d'argile durant de courtes périodes réductrices au cours de l'année.

Un mouvement de fer $\left(I_{\mathrm{fc}}=2,8\right)$ et d'argile $\left(\mathrm{I}_{\mathrm{a}}=1,4\right)$ s'opère dans le sol brun faiblement lessivé. Les conditions de station favorisent dans ce type une amorce d'altération du matériau pédologique. L’hydromorphie modérée, qui provient de la circulation imparfaite des eaux au-dessus d'un substratum imperméable (burdigalien) affecte la base du pédon. Par ailleurs, des phénomènes de microgleyification encore discrets qui se produisent au cours de lannée ont tendance à "dégrader * les horizons superfiiciels, mais l'activité biologique reste très satisfaisante. 
Le fer amorphe augmente sensiblement vers la base du profil et le maximum de fer amorphe coïncide avec le maximum du rapport Feo/Fed. Ce fait peut s'expliquer d'une part par un taux élevé de libération du fer en $\mathrm{B}$ et $\mathrm{C}$ qui intervient dans un matériel acide, d’autre part par une migration préférentielle des amorphes en profondeur.

Le rapport Alo/Ald augmente nettement dans les horizons $B$, ce qui comme pour le fer, indique labondance des formes amorphes.

Les rapports Fed/argile et Ald/argile restent sensiblement constants dans ces sols.

\subsection{Les critères biochimiques}

Nous avons cherché à inventorier les types d'humus ${ }^{(2)}$, profil par profil, et à préciser la nature et le rôle des complexes organo-minéraux.

\subsection{La matière organique}

En milieu frais, la forêt feuillue induit la formation d'un humus biologiquement actif $(C / N \leqslant 16)$, aéré et bien structuré.

L'horizon Al montre, en lumière naturelle, une matière organique fine abondante, éparpillée dans la masse minérale sous forme d'une multitude de boulettes fécales noires accolées aux grains de quartz et aux minéraux colorés. L'assemblage s'organise en petits agrégats disposés de façon très lâche les uns par rapport aux autres. Les débris végétaux. micro-fragmentés, sont en quantité limitées.

Le rapport des fractions fines et grossières (Carbone $F<50 \mu \mathrm{m} /$ Carbone $\mathrm{F}>100 \mu \mathrm{m})$ dépasse toujours l'unité et reste inférieur à 1,5.

La plus grande partie du carbone (plus des 3/4) reste inextractible : les humines prédominent largement dans ce type d'humus (cf. tabl. 5). Le tétraborate de sodium et la soude exercent un pouvoir complexant sensiblement équivalent (\# 40 p. 100 du carbone extrait). Les acides fulviques lemportent nettement sur les acides humiques $(\mathrm{AF} / \mathrm{AH}>2)$.

Dans les milieux "secs . la couverture végétale acidifiante donne naissance à des dysmoders souvent minces (de l'ordre de 5 ou $6 \mathrm{~cm}$ ) ou, à un stade plus évolué, à des mors sensiblement plus épais $(>10 \mathrm{~cm})$. L’accumulation de restes végétaux à la surface du sol et le $\mathrm{C} / \mathrm{N}$ très élevé $(\geqslant 24)$ que l'on enregistre en $\mathrm{Al}$ indiquent une qualité édaphique de ces stations nettement moins bonne. Ces humus déclenchent des phénomènes de micro-podzolisation.

L'horizon Al est composé de matière organique fraîche intensément fragmentée et de constituants végétaux fortement altérés, en voie de transformation avancée, «piquetés» de multiples cristaux de quartz. On observe par endroits des plages de matière fine aggrégée.

La majorité du carbone est répartie dans la fraction supérieure à $100 \mu \mathrm{m}$. Les rapports Carbone $\mathrm{F}<50 \mu \mathrm{m} /$ Carbone $\mathrm{F}>100 \mu \mathrm{m}$ restent toujours inférieurs à l'unité et tendent vers 0,5 .

(2) De manière à caractériser les humus, nous avons été amenés à multiplier les échantillons dans chaque milié considéré. 


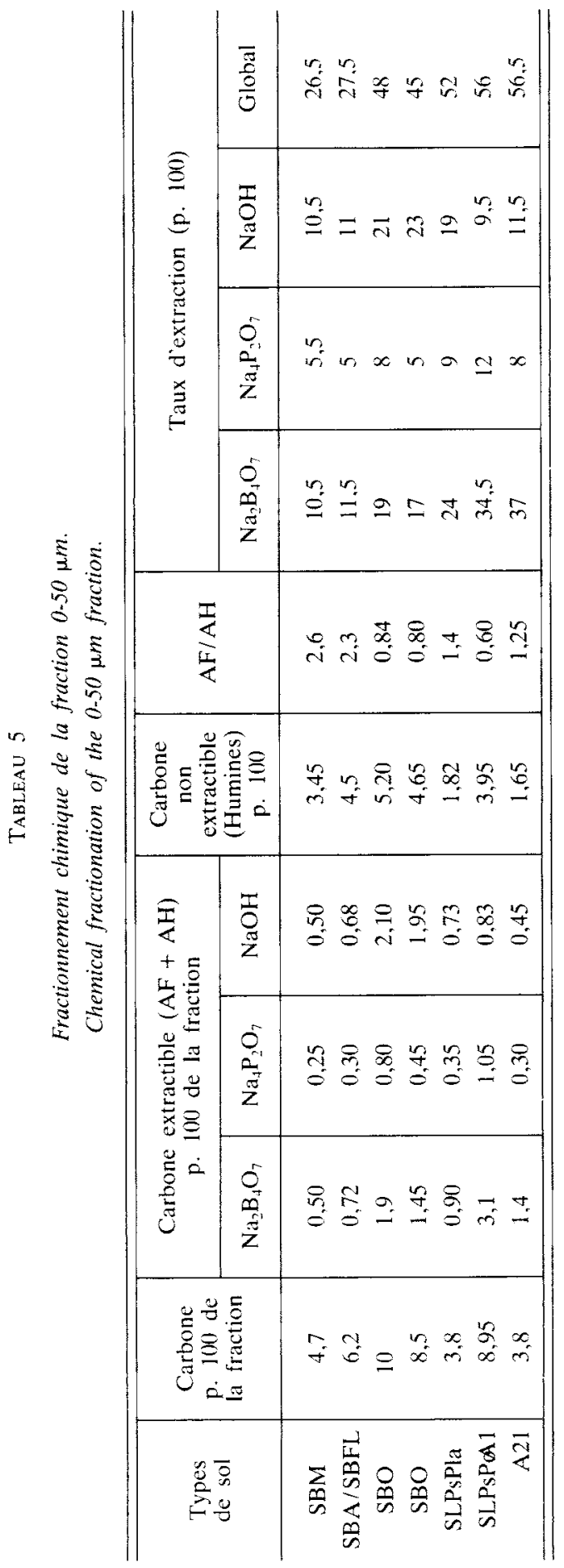


Les taux d'extraction obtenus avec le tétraborate et la soude sont très élévés, mais laction de ce dernier réactif prime, d'autant plus nettement que le ralentissement de l'activité biologique est plus prononcé. Les carbones extractible et inextractible ont tendance à s' " équilibrer », les humines restant cependant majoritaires. Les rapports $\mathrm{AF} / \mathrm{AH}$ avoisinent 0.8 dans les deux sols bruns ocreux, ce qui traduit la migration des acides fulviques dans les horizons de profondeur.

Dans les zones hydromorphes, l'action de la nappe provoque la formation de variantes de type hydromoder et hydromor. Le fait marquant réside dans l'importance de la matière fine : le rapport Carbone $\mathrm{F}<50 \mu \mathrm{m} /$ Carbone $\mathrm{F}>100 \mu \mathrm{m}$ dépasse toujours 1,5 en $\mathrm{A} 1$ et tend vers 2. Ce rapport semble augmenter avec l'intensité de I'hydromorphie.

Dans l'hydromoder coexistent une matière organique fraîche, intensément fragmentée, très abondante, disséminée dans la masse minérale, des débris végétaux fortement altérés piquetés de multiples paillettes de quartz et de nombreuses boulettes humiques noir foncé isolées dans le squelette minéral.

L'hydromor se caractérise par un système d'assemblage où se juxtaposent des grains minéraux, des boulettes fécales et de la matière fine. L'ensemble matière organique-matière minérale apparaît plus compact et les espaces créés par le mode d'organisation du matériau semblent trop limités pour que se réalisent une activité de la faune et une circulation de l'air et de l'eau satisfaisantes.

Le tétraborate constitue un agent complexant très efficace, capable de solubiliser 35 p. 100 du carbone de l'échantillon en Al. Au total, les trois réactifs utilisés successivement extraient plus de 50 p. 100 du carbone. Les acides fulviques, résistants à la biodégradation, migrent en profondeur, en particulier dans le sol lessivé hydromorphe podzolique où le rapport $\mathrm{AF} / \mathrm{AH}$ égal à 0,6 en $\mathrm{Al}$ remonte sensiblement en A21 $(1,25)$.

TABleaU 6

Analyse des formes organo-complexées : indices pédogénétiques *.

Analysis of organo-mineral associations : pedogenetic indexes *.

\begin{tabular}{c|l|c|c|c|c|c}
\hline $\begin{array}{c}\text { Type } \\
\text { de sols }\end{array}$ & Horizon & Fet/Fed & Alt/Ald & Fep/Fed & Fet/Fep & Alt/Alp \\
\hline \multirow{2}{*}{ SBM } & A1 & 0,1 & 0,2 & 0,4 & 0,27 & 0,37 \\
& A1 (B) & 0,1 & 0,2 & 0,4 & 0,27 & $\mathbf{0 , 3 7}$ \\
SBA/SBFL & A1 & 0,1 & 0,2 & 0,4 & 0,3 & 0,3 \\
& A2 (B) & 0,12 & 0,2 & 0,4 & 0,3 & $\mathbf{0 , 3}$ \\
\hline SBO & A1 & 0,25 & 0,8 & 0,42 & 0,6 & 1 \\
(Nouan) & A1Bh & 0,25 & $\mathbf{0 , 8}$ & 0,58 & 0,43 & $\mathbf{0 , 6 5}$ \\
SBO & A1 & 0,2 & 1 & 0,42 & 0,5 & 1 \\
(La Saulot) & A2Bh & 0,2 & 1 & 0,5 & 0,4 & 0,8 \\
\hline \multirow{2}{*}{ SLPsPla } & A1 & 0,26 & 0,8 & 0,93 & 0,28 & 0,8 \\
& A21 (g) & 0,13 & $\mathbf{0 , 8}$ & 0,53 & 0,25 & $\mathbf{0 , 8}$ \\
SLPsPo & A1 & 0,53 & 0,83 & 0,8 & 0,65 & $\mathbf{0 , 8 3}$ \\
& A21 & 0,60 & $\mathbf{0 , 8 7}$ & 0,8 & 0,76 & $\mathbf{0 , 8 7}$ \\
\hline
\end{tabular}

* Rapports atomiques des quantités de fer et d'aluminium extraites par le tétraborate de sodium (Fet, Alt), le pyrophosphate de sodium (Fep. Alp) et le « dithionite-citrate-bicarbonate » de sodium (Fed, Ald). 


\subsection{Les complexes organo-minéraux}

Le degré de complexation du fer et de l'aluminium par les ligands organiques est un bon indice de podzolisation commençante. La tendance générale dans les profils étudiés va dans le sens d'un accroissement des formes organo-complexées dans les horizons diagnostiques des sols bruns acides aux sols bruns ocreux. Un constat analogue peut être fait pour les sols lessivés hydromorphes: le degré de séquestration des éléments métalliques s'accentue dans les profils affectés par la dégradation ou la podzolisation.

Dans les sols brunifiés qui se développent dans les milieux frais, les indices pédogénétiques qualitatifs (cf. tabl. 6) restent faibles $(<0,5)$. Ces rapports et, plus spécialement Alt/Ald et Alt/Alp, s'élèvent sensiblement dans les sols marqués par la podzolisation modérée en milieu «sec ». Dans les zones hydromorphes, les quantités organo-complexées semblent importantes, surtout pour l'aluminium.

\section{Caractérisation des séquences évolutives spécifique de chaque milieu et mode de répartition des sols dans le paysage}

Les profils répertoriés dans les trois grands milieux biophysiques varient, en fonction des conditions de station, à l'intérieur d'une séquence pédogénétique caractéristique depuis des profils stables, peu différenciés, en équilibre avec les facteurs édaphoclimatiques vers des sols marqués de façon croissante par l'acidification, la podzolisation, l'hydromorphie ou la dégradation.

\subsection{Les zones hydromorphes}

La géométrie des corps sédimentaires et leur extension conditionnent étroitement la répartition des sols influencés par l'hydromorphie. La grande variabilité pédologique est due à la grande diversité des facteurs stationnels (caractéristiques de matériau, microtopographie, importance de la couverture pédologique, dynamique de la nappe et pédoclimat) qui multiplie les processus pédogénétiques et les modèles évolutifs (lessivage, hydromorphie, planosolisation, glossification, dégradation, aliotisation, podzolisation). La distribution spatiale des groupements végétaux (bétulaie à mousses et germandrée, bétulaie à chèvrefeuille, ronces et mousses, bétulaie-saulaie-chênaie à ronces, mousses et joncs...) reflète bien les nuances pédogénétiques: les équilibres sol-végétation correspondent globalement à des paraclimax stationnels.

Les sols qui se développent dans les zones hydromorphes, outre les variantes pseudogley (modal, podzolique ou planosolique) et gley (alluviaux, rarement humiques), s’intègrent dans la sériation évolutive suivante : sol brun lessivé $\rightarrow$ sol lessivé planosolique $\rightarrow$ sol lessivé glossique $\rightarrow$ sol lessivé podzolique $\rightarrow$ sol dégradé hydromorphe. Sur un plan minéralogique, les argiles les plus caractéristiques sont, en dehors des micas, les smectites, la kaolinite, la vermiculite, et la lépidocrocite.

Ces sols génétiquement non liés s'imbriquent dans le paysage : les conditions de stations éminemment variables sont responsables de juxtapositions de sols sensiblement différents et difficiles à cartographier (mosaïques). 


\subsection{Les milieux secs}

Le processus pédogénétique qui a commandé la formation des sols sur les sables remaniés des interfluves est la brunification. Mais, l'homme, en bouleversant l'équilibre naturel de la région (Allorge \& Gaume, 1925). a profondément modifié les données biologiques, de sorte que la végétation exerce, dans les milieux "secs", une action esssentielle dans la différenciation et l'évolution pédogénétiques (GuELlEC, 1980).

Les bétulaies claires accompagnées de graminées et de germandrée, qui représentent un stade intermédiaire entre les formations primitives et les peuplements secondaires actuels, se développent sur des sols bruns, parfois légèrement lessivés, peu colorés et pauvres en matière organique. Ces formations n'existent qu'à l'état de lambeaux et ce sont des peuplements dégradés (bétulaies à Ericacées, à lichens, chênaies claires à callune) et surtout des phases de végétation nouvelles (landes boisées à pins et bouleaux), constituant en quelque sorte un disclimax, que l'on rencontre le plus souvent. Avec la dégradation de la végétation va de pair une évolution régressive des sols, mais ce phénomène, dans la plupart des situations, ne fait encore que démarrer. Par rapport au sol brun acide type, les profils actuels marquent fréquemment un début d'altération comme le montrent certains caractères de la matière organique $(\mathrm{C} / \mathrm{N}, \mathrm{AF} / \mathrm{AH})$, la nature du complexe absorbant $\left(\mathrm{H}^{+} / \mathrm{A} 1^{3+}, \mathrm{S} / \mathrm{Te}\right)$ et, dans les types les plus évolués, la transformation morphologique des pédons (structure plus dispersée, couleur plus vive), la mobilisation des éléments métalliques et la fréquence de micropodzols $(<15 \mathrm{~cm})$.

Les sols brunifiés plus ou moins évolués et les intergrades podzoliques (sol brun ocreux) couvrent de grandes surfaces en Sologne et constituent le type pédogénétique le plus caractéristique des milieux «secs». Les sols ocre-podzoliques et podzoliques bien développés sont en effet plus localisés, liés à des conditions de station particulièrement sévères (matériau très quartzeux dépourvu de limons, d’argile et de minéraux altérables, couverture végétale acidifiante plus ancienne). Le plus souvent, l'évolution pédologique, sur les sables remaniés de la "Sologne des étangs", n'a pas dépassé le stade du sol faiblement podzolique. Il faut noter, à ce propos, l'absence de sols franchement lessivés, sous la lande boisée ou sous résineux. Le type sol lessivé podzolique manque le plus souvent dans ce milieu: le processus de podzolisation modérée se déclenche, semble-t-il, alors même que la dispersion et la mobilisation de l'argile ne fait que s'amorcer. Des observations similaires ont été faites récemment (Herbauts, 1981 et 1982) sur les profils développés sur les sables d'altération des calcaires gréseux en Lorraine belge.

La séquence évolutive en milieu « $\mathrm{sec}$ " peut être schématisée de la façon suivante : sol brun acide $\rightarrow$ sol brun ocreux $\rightarrow$ sol ocre-podzolique $\rightarrow$ sol podzolique. La fraction fine est représentée par des micas, par la kaolinite, un peu de vermiculite et de lépidocrocite.

\subsection{Les milieux frais}

La brunification est le processus pédogénétique majeur qui caractérise les profils de bas de versants. Le matériau intervient de manière décisive dans l'orientation de la pédogenèse et joue un rôle moteur dans la dynamique évolutive de ces milieux : de sa teneur en argile ou en limons et en minéraux altérables, de ses propriétés physiques (aération) dépendent le type d'altération et l'intensité de l'activité biologique. 
Le lessivage reste un phénomène secondaire qui se surimpose le plus souvent au processus général de la brunification d'autant que la topographie doucement inclinée en amont tend, par entraînement oblique des éléments, à " effacer " ses effets.

Les sols brunifiés sains caractéristiques des milieux frais occupent des surfaces relativement restreintes mais n'en constituent pas moins un élément constant du paysage solognot. De par les conditions de station, l'équilibre de ces écosystèmes forestiers relativement peu perturbés par l'homme apparaît éminemment fragile. Certains profils sont affectés par l'hydromorphie modérée, qui se trouve parfois favorisée par la topographie (surfaces subhorizontales cloisonnées), et subissent les effets d'une altération plus poussée.

Les sols caractéristiques de ces milieux relativement homogènes s'inscrivent dans la série évolutive suivante ${ }^{(3)}$ : sol brun mésotrophe ou acide $\rightarrow$ sol brun faiblement lessivé $\rightarrow$ sol brun lessivé $\rightarrow$ sol brun ou brun lessivé marmorisé $\rightarrow$ sol brun ou brun lessivé hydromorphe. Ce dernier type fait transition avec des zones hydromorphes. Les minéraux argileux sont essentiellement constitués de micas. Ceux-ci sont accompagnés de petites quantités de kaolinite et de lépidocrocite.

Les chênaies acidiphiles, les chênaies claires à germandrée et les chênaies à canche s'installent sur les sols chimiquement les plus riches, mais les chênaies à fougère-aigle se développent sur des sols sensiblement plus profonds et possédant de meilleures réserves en eau.

Malgré la grande hétérogénéité qui existe parfois à l'échelle de la parcelle (dépressions hydromorphes "perchées " sur les interfluves, milieux "secs » installés sur les points bas de la topographie où de faibles différences de niveau ( 1 ou $2 \mathrm{~m}$ ) suffisent pour induire des types de pédogenèse différents) les types de sédiments mis en place respectent un certain ordonnancement à l'échelle locale ou régionale et sont responsables de nuances sensibles dans le paysage actuel qui se répètent plus ou moins régulièrement. La topographie, qui gouverne en premier lieu le pédoclimat, le type de matériau et la végétation agissent dans le même sens et ajoutent leurs effets pour diversifier un milieu qui pouvait paraître à première vue monotone.

\section{Conclusion}

L'étude de la genèse et de l'évolution des sols développés sur la formation de Sologne et sur les sables issus de son remaniement a été l'objectif essentiel de ce travail. Deux grandes étapes ont marqué cette recherche sur la pédogenèse acide sur sables: dans l'approche écologique, nous avons reconnu les facteurs principaux du déterminisme pédogénétique, le matériau essentiellement, puis le pédoclimat et la végétation, qui sont des données "secondes", et la topographie. Les analyses de caractérisation réalisées sur un échantillonnage restreint mais sélectif ont fourni les éléments fondamentaux de la nature des sols et ont permis de donner une définition précise des types pédogénétiques les plus caractéristiques de la «Sologne des étangs ". Nous sommes ainsi passés de l'observation écologique d'un ensemble de profils repré-

(3) Un faciès particulier, le sol brun lessivé à illuviation en bandes, encore discrètes, a été observé sur des bourrelets alluviaux sableux à sablo-limoneux. 
sentatifs à l'étude de l'évolution comparée du «climat d'altération » des sols des trois milieux biophysiques considérés et avons essayé d’établir un lien logique entre l'écologie et les orientations de la pédogenèse.

Plusieurs phénomènes majeurs ont joué un rôle décisif dans la genèse, dans la répartition et dans la dynamique évolutive des sols en Sologne. Ce sont :

- La mise en place durant le Burdigalien et l'Helvétien de matériaux aux caractéristiques mécaniques et chimiques plus ou moins contrastées et présentant des extensions dans l'espace éminemment variables.

- L'existence de périodes mécaniques de remaniement et d’apport, qui ont été favorisées par la disparition du couvert végétal au cours des périodes froides du quaternaire. Ce processus géomorphologique a légué un matériau de couverture (sables remaniés) qui fossilise complètement ou partiellement le substratum miocène ou les horizons paléosoliques.

- L'existence de phases érosives au cours de l'Holocène qui ont modelé le paysage solognot actuel.

- Des transformations historico-géographiques du couvert végétal qui ont rompu l'équilibre biophysique de la région.

Les milicux "secs» se localisent sur les sables remaniés qui ont été épargnés par l'érosion et parfois quelque peu « retouchés " par des apports ultérieurs.

Les zones hydromorphes s'installent sur les affleurements, essentiellement sabloargileux de la formation de Sologne.

Les milieux frais, qui se développent, comme les milieux secs, sur les sables remaniés se différencient outre la végétation par des conditions de topographie (bas de versants), de pédoclimat, et souvent de matériau (enrichissement par colluvionnement).

Toutes les données analytiques fines (critères chimiques et biochimiques) convergent et confirment le diagnostic de la macro-analyse (critères texturaux, données biologiques, caractères pédoclimatiques) réalisé au cours du travail de prospection approfondi.

Les associations forestières reflètent bien les conditions de station qui prévalent en Sologne, en particulier dans les zones hydromorphes et les milieux frais. Dans les milieux «secs", la végétation est globalement un bon indicateur du type de développement pédogénétique, mais, du fait de la jeunesse relative du matériel solognot et du bouleversement récent de la couverture végétale engendré par l'action de l'Homme. des discordances sol-végétation existent également.

Reçu le 10 septembre 1984. Accepté le 8 avril 1987. 


\section{Remerciements}

Jexprime ma reconnaissance à Messieurs Aguesse et Lelong, respectivement Professeurs d'Ecologie et d’Hydrogéologie à l'Université d'Orléans-La-Source et à Monsicur Souchier, Professeur à l'Université de Grenoble et ancien Directeur du Centre de Pédologie du C.N.R.S. de Vandouvre.

Je remercic tout particulièrement Messicurs Jeanroy et Rouiller du Centre de Pédologic ct Monsicur Ghio, Chef de la Division Gćologie du Sédimentaire au B.R.G.M.

\section{Summary}

Characterization of some major soils of Sologne environment. Influence of site factors on the distribution and the evolution of profiles.

The scope of this work is to characterize and to assess the development sequences of forest soils formed on the Sologne geologic formation and on the superficial sands coming from their alteration and deposition during the glacial stages of the Pleistocene. Three major pedogenetic zones have heen recognized:

The « dry » areas settled on removed sands, where the vegetation exert an essential role on the diversification of profiles, inducing an incipient podzolisation.

The hydromorphic zones developed on the outcrops of Sologne formation, in which the site conditions are responsible of associations of soils marked by increasingly leaching, mottling or degradation processes.

The fresh lands localized on the lower slopes, characterized by specific vegetation, pedoclimate and sometimes material data.

The analytical methods used in this work lead to the same conclusion and justify the biophysical divisions of the Sologne landscape. The criteria utilized are as follows:

- The distribution pattern of clay. Depletion of $A$ horizons of clay and concentration in $B$ horizons are an outstanding feature of "hydromorphic" soils (see paragraphe 5.1.). On the opposite, translocation of clay is always a minor process in the other arcas.

- The complex exchange. Sediments occuring in Sologne are derived form the same geochemical province (Massif Central), so the cation saturation ratio and the percentage base saturation depends directly on site factors. In particular, the $\mathrm{Ca} / \mathrm{Mg}$ ratio and the exchange acidity $\left(\mathrm{H}^{+}+\right.$ $\mathrm{Al}^{31}$ ) discriminate significantly the zones distinguished (see paragraph 5.2. and tabl. 3).

- The vertical distribution of oxalate and dithionite extractible iron and aluminium and of qualitative ratio $\left(\mathrm{M}^{+} \mathrm{o} / \mathrm{M}^{+} \mathrm{d}, \mathrm{M}^{+} / \mathrm{clay}, \mathrm{Feo} / \mathrm{Alo}\right)$ as shown in figure 1 and table 4 .

- The physical $(\mathrm{F}<50 \mu \mathrm{m} / \mathrm{F}>100 \mu \mathrm{m})$ and chemical (AF/AH, extraction ratcs) fractionnation of organic matter and the analyse of the alkali-soluble organo-mincral compounds.

In spite of the great variability of soils frequently observed even in small areas, the spatial arrangement of sediments determine, at local or regional scales, a particular soil distribution pattern.

The pedogenetic routes are strongly related to the forest associations, so that the soilvegetation combination constitute a useful key for recognizing and classifying forest sites.

Key words: Soil characterization, soil vegetation, relationships in Sologne. 


\section{Références bibliographiques}

Allorge A., Gaume R., 1925. Bull. Soc. Bot. Fr, 72, 5-59.

Ameryckx J., 1960. Pédologie, 10 (1), 124-190.

Aubert G., 1974. Cours D.E.A.

Blume H.P., Schwertmann U., 1969. Soil Sci. Soc. Am. Proc., 33, 438-444.

Braun-Blanquet J., 1967. An. Edapt. Agrobio. Madrid, 53-87.

Buol S.W., 1973. So. Coop. Ser. Bull., 174, U.S.D.A.

Burtin G., Roulller J., Souchier B., 1972. Bull. E.N.S.A.I.A. Nancy, 14 (2), 193-205.

Coen G.M., Pawluk S., 1966. Can. J. Soil. Sci, 46, 245-254.

De Coninck F., Laruelle J., 1964. Soil Micromorphology, Elsevier Publ. Cy, 169-188.

De Coninck F., Herbillon A., 1969. Pédologie, 19, 159-272.

Dupont, Servant, 1983. Carte pédologique au 1/50000 de Vierzon (Cher).

Folks H.C., Riecken F.F., 1956. Soil. Sci. Soc. Am. Proc., 20, 575-580.

Gaussen H., 1955. C.R.H. Acad. Sc., 240, 642-644.

Gigout M., Horemans P., Rasplus L., 1972. Bull. B.R.G.M., 1 (1), 1-28.

Godron M., 1964. Carte phytoécologique et carte de l'occupation des terres en Sologne. Feuilles Argent/Sauldre 2 Sud et 5 Sud au 1/10000. Notice détaillée. C.E.P.E. Montpellier.

Gras J., 1963. Thèse Doc. Etat. Paris, $494 \mathrm{p}$

Guellec I., 1980. Compte rendu D.G.R.S.T., 13-21.

Guellec I., 1980. Bull. Inst. Eco. Appl., Orléans, 3-4, $29-45$.

Gueillec I., 1982. Recherches éco-pédologiques en Sologne centrale. Caractérisation des types majeurs évolutifs de sol. Document non publié.

Herbauts J., 1981. Science du Sol, 3, 187-217.

Herbauts J., 1982. Journal of Soil Science, 33 (4), 743-762.

Horemans P., 1961. Thèse Doc. $3^{e}$ cycle, Paris, 164 p.

Juste C., 1965. Thèse Doc. Ing., Nancy, 142 p.

Kundler P., 1961. Arch. für Forstwesen, 10, 451-469.

Le Houerou H.N., 1961. D.E.S., Montpellier, 73 p.

Lelong F., Pich P., 1978. Sciences de la Terre, 22 (3), 243-271.

Musset R., 1943. Ann. Géogr.

Racineux, 1983. Carte pédologique au 1/50 000 de Vierzon (Loir et Cher).

Rasplus L., 1978. Thèse Doc. Etat, Orléans.

Ricardo H., 1971. Thèse Doc. $3^{\text {e }}$ cycle, Paris.

Robin A.M., 1979. Thèse Doc. Etat, Nancy, 173 p.

Studer, Servant, 1981. Carte pédologique au 1/50000 de la Feuille de Léré.

Studer, 1983. Carte pédologique au 1/50000 de Montrichard.

Wuerman E., Whiteside E.P., Mortland M.M., 1959. Soil. Sci. Soc. Am. Proc., 23, 135-143. 